\title{
PALEOECOLOGÍA MEDIANTE FORAMINÍFEROS DEL PALEÓGENO DEL ÁREA DORADO SUR, DE LA CUENCA DE MAGALLANES, CHILE
}

\author{
PALEOECOLOGY BY USING PALEOCENE FORAMINIFERA FROM \\ DORADO SUR AREA OF THE MAGELLAN BASIN, CHILE
}

Margarita Marchant ${ }^{1}$

\begin{abstract}
We analyzed paleogene foraminifera between 10 and $2.287 \mathrm{~m}$ depth from samples taken from the oil well of hydrocarbon extraction, Dorado Sur $\mathrm{N}^{\circ} 1$, localized in the continental margin of the northwestern sector of Santiago Bay, Magellan Strait in order to reconstruct the paleoecology. The analysis of the faunal composition allows distinguishing two ingressions: one for the Brunswickian-Clarencian of early Eocene-Middle Eocene, and the other in the Cameronian of Late Eocene age, recorded in both outer shelf waters. Instead on floors Germanian, Moritzian, Rosarian-Gaviotian and Miradorian recorded inner shelf environments.
\end{abstract}

Key words: Paleoecology, Foraminifera, Paleogene, Magallanes Basin, Chile.

\section{RESUMEN}

Se analizaron los foraminíferos del Paleógeno entre 10 y 2.287 m de profundidad, provenientes de muestras obtenidas del pozo de extracción de hidrocarburos, Dorado Sur $\mathrm{N}^{\circ} 1$, ubicado en el sector continental al noreste de la Bahía Santiago, Estrecho de Magallanes, con el objeto de reconstruir su paleoecología. El análisis de la composición faunística permitió reconocer dos ingresiones: una durante el Brunswickiano - Clarenciano, de edad eocena temprana - eocena media, y la otra en el Cameroniano de edad eocena tardía, registrándose, en ambas, aguas de plataforma externa. En cambio en los pisos Germaniano, Moritziano, Rosariano-Miradoriano y Gaviotiano se registraron ambientes de plataforma interna.

Palabras clave: Paleoecología, Foraminíferos, Paleógeno, Cuenca de Magallanes, Chile.

1 Departamento de Zoología, Universidad de Concepción, Casilla 160-C, Concepción, Chile. 


\section{INTRODUCCIÓN}

Los trabajos micropaleontológicos del Paleógeno de Chile son escasos, debido principalmente a la falta de herramientas estratigráficas capaces de proporcionar validez a los resultados.

La primera zonación del Paleógeno de Chile Central, mediante foraminíferos planctónicos fue realizada por Martínez (1968). Por otra parte, los foraminíferos de la cuenca de Magallanes (parte chilena), no se conocen en forma exhaustiva. Así, las asignaciones de edades de las distintas unidades estratigráficas están basadas principalmente en foraminíferos bentónicos, debido a la pobre representación de foraminíferos planctónicos durante el Paleógeno de la cuenca de Magallanes (Cañón en Natland et al. 1974).

La investigación micropaleontológica de la cuenca de Magallanes se puede resumir como sigue: los trabajos de Todd \& Kniker (1952) y Natland \& González (1965), propusieron el sistema de pisos para la cuenca de Magallanes, actualizado posteriormente por Cañón (1968a y b), Natland et al. (1974) y Cañón \& Ernst (1975) en Hromic 1990), así como por varios autores a través de estudios estratigráficos realizados por instituciones nacionales (DEMIPE, CORFO y ENAP en Natland et al. 1974). Finalmente, Marchant (1998) estableció una doble sucesión cronoestratigráfica del Paleógeno. La primera de ellas se basa en foraminíferos bentónicos y la segunda mediante foraminíferos planctónicos asignando las edades de las distintas unidades estratigráficas del área Dorado Sur-Kimiri Aike Sur de la cuenca de Magallanes.

En el presente trabajo se pretende reconstruir las condiciones paleoecológicas de sedimentación en el área Dorado Sur, mediante foraminíferos bentónicos principalmente. Para lo cual consideraremos que:

- Los foraminíferos, en general, son casi exclusivamente organismos marinos y presentan una amplia distribución, tanto batimétrica como geográfica. Este hecho, así como sus características biológicas, explican por qué desempeñan un rol importante en los estudios oceanográficos (Boltovskoy 1965).

- Las especies del pasado geológico pueden ser interpretadas con asociaciones similares a las actuales, las cuales determinan ambientes tanto en temperatura, profundidad como en los sedimentos. Este concepto se utiliza para interpretar biofacies de acuerdo a probables profundidades a través de Homeomorfos, es decir especies similares y emparentadas (Bandy \& Arnal 1960).

- Los datos paleoecológicos se han recopilado principalmente de los siguientes trabajos: Todd \& Kniker (1952); Bandy \& Arnal (1960); Boltovskoy (1965); Cañón (1968a, b); Natland et al. (1974), Malumián (1978). El esquema batimétrico utilizado es el entregado por Boltovskoy (1965) y que se detalla a continuación:

I.- Zona Intermareal: esta zona está situada entre los niveles de pleamar y bajamar. Es una zona de cambios muy bruscos de las condiciones ecológicas, con movimientos de agua con diferente carácter y fuerza. Los foraminíferos de esta zona son pequeños, achatados y se encuentran generalmente adosados al sustrato. Son característicos los géneros Discorbis, Trochammina, Elphidium y Quinqueloculina.

II.- Zona Sublitoral: comprende desde el nivel de baja mar hasta el talud, es decir, la zona de la plataforma continental. Comúnmente se la divide en zona de turbulencia, plataforma interna y plataforma externa.

a) Zona de Turbulencia: corresponde al área donde tienen lugar los movimientos del agua causados por tormentas, mareas, resacas, etc. Su profundidad promedio es de $20 \mathrm{~m}$ y le son propios los foraminíferos con paredes gruesas y fuertes o con el caparazón adherido al sustrato. Muchos de ellos también se hallan en la zona intermareal. Son características las especies de los géneros Elphidium, Rotalia, Quinqueloculina, Poroeponides y a menudo Textularia.

b) Plataforma Interna: zona que alcanza profundidades entre los 50 y $70 \mathrm{~m}$; la fauna de foraminíferos se hace visiblemente más variada y más rica cuantitativamente. Las especies aglutinadas constituyen un porcentaje relativamente pequeño y están representadas principalmente por géneros primitivos, e.g. Textularia, Trochammina, Reophax y Saccammina. Las especies planctónicas se encuen- 
tran excepcionalmente y sólo en aquellos lugares donde la plataforma continental es estrecha y el aumento de la profundidad es brusco. Al grupo de géneros dominantes, aparte de Rotalia, Elphidium, Quinqueloculina, se agregan algunos representantes de las familias Miliolidae, Lagenidae y Buliminidae.

c) Plataforma Externa: alcanza profundidades entre 70 y 200 m. Esta zona tiene mucho en común con la precedente, aunque existen ciertas diferencias: i) el número de géneros y especies es visiblemente mayor, el doble en promedio, ii) el porcentaje de ejemplares aglutinados es notoriamente menor, iii) el porcentaje de ejemplares de foraminíferos planctónicos aumenta, hasta que, en el margen de esta zona llegan a predominar sobre los bentónicos, iv) aumenta la importancia de las especies hialinas de las familias Lagenidae, Buliminidae, del género Cibicides y de algunos otros, a expensas de las especies porcelanáceas, cuyo número decrece (excepto Pyrgo, que se hace más numeroso). Como géneros predominantes se pueden mencionar dentro de los calcáreos a Cassidulina, Cibicides, Nonion, Nonionella, Uvigerina y Virgulina y dentro de los aglutinados a Bigenerina y Martinotiella.

III.- Zona Batial: esta zona comienza con el límite inferior de la plataforma continental y finaliza ca. de los $3.000 \mathrm{~m}$ de profundidad. Phleger (1960 fide Boltovskoy 1965) distingue:

a) Talud Superior (200-1.000 m): el número de géneros y especies de foraminíferos bentónicos no varía mucho, pero aumenta considerablemente la relación planctónico-bentónica siendo mayor a la unidad. La cantidad de ejemplares aglutinados sigue constituyendo alrededor del 5\% del total. Si bien continúa la misma tendencia de predominio de las especies grandes y de construcción complicada, en algunos lugares se pueden encontrar casos en que las formas pequeñas y de estructura simple son las más numerosas. Los géneros dominantes son Bolivina, Uvigerina, Cassidulina, Gyroidina, Chillostomella, Allomorphina, Pullenia y Bulimina. Entre los porcelanáceos tiene gran importancia el género Pyrgo.

b) Talud Inferior (más de 1.000 m): esta zona se caracteriza por la disminución, tanto cualitativa como cuantitativa, de la fauna de foraminíferos bentónicos. De acuerdo con esto, la relación planctónico-bentónica aumenta y llega hasta 10 o más. En la población bentónica aumenta el porcentaje de especies aglutinadas. Los géneros más comunes son Bulimina, Epistominella, Glomospira, Gyroidina y Pullenia.

IV.- Mar Profundo: aumenta el porcentaje de formas aglutinadas: Technitella, Cyclammina, Thurammina y Astrorhizidae, típicas de aguas profundas. La Familia Miliolidae a esta profundidad presenta caparazón, formado por una capa de sílice homogénea (debido a la compensación del carbonato).

Finalmente, es conveniente decir que es el conjunto faunístico en su totalidad el que definirá y limitará el ambiente paleoecológico. No obstante, este análisis se basará principalmente en los foraminíferos bentónicos, ya que son buenos indicadores para descifrar y reconstruir detalles del paleoambiente (Boltovskoy 1980b).

\section{MATERIAL Y MÉTODOS}

Se estudiaron los foraminíferos planctónicos y bentónicos del pozo petrolífero Dorado Sur $\mathrm{N}^{\circ}$ 1 , denominado de aquí en adelante como DS1, entre los 10 y $2.287 \mathrm{~m}$ (Fig. 1). Este pozo DS1 se encuentra ubicado en la parte continental, entre la Primera y Segunda Angostura del Estrecho de Magallanes, Chile, específicamente en el sector ubicado inmediatamente al noroeste de la Bahía Santiago $\left(52^{\circ} 30^{\prime} \mathrm{S} ; 7^{\circ} \mathrm{W}\right)$ (Fig. 1). Las muestras provienen de "cutting", cada nueve metros (Tabla 1). La datación de los pisos analizados corresponde a Marchant (1998).

Tabla 1. Detalle de las muestras del Pozo Dorado Sur №1 (PD1). Se incluye el número de muestras $(\mathrm{N})$ por categoría.

\begin{tabular}{lrrc}
\hline \multicolumn{1}{c}{ Carta } & \multicolumn{2}{c}{ DS1 } & $\mathrm{N}$ \\
\hline Cota M. R. (m) & 10 & Estériles & 40 \\
Cota Terr. (m) & 6,5 & Productivas & 82 \\
Latitud & 4.178 .883 & Total & 122 \\
Longitud & 432.268 & & \\
Prof. max. (m) & 2.287 & & \\
Est. Microp. & J. Sillard & & \\
Año & 1975 & & \\
Clasificación & Yac. 1619 & & \\
\hline
\end{tabular}




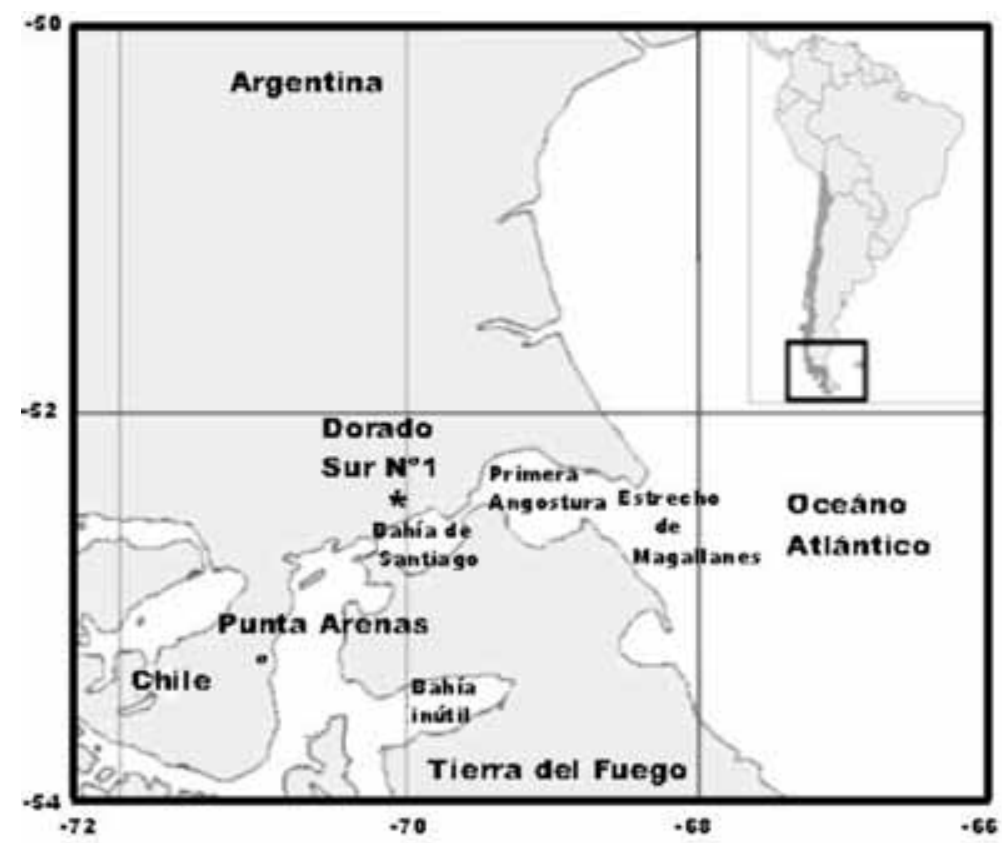

Fig. 1. Ubicación geográfica del área de estudio, pozo Dorado Sur №1 (").

El material pertenece a la colección micropaleontológica de la Empresa Nacional de Petróleo (ENAP-Magallanes), que está depositada en el Laboratorio de Micropaleontología del Instituto de la Patagonia, Universidad de Magallanes, Punta Arenas, Chile.

La clasificación genérica utilizada corresponde a la de Loeblich \& Tappan (1988). La determinación específica y los rangos estratigráficos se basan en la descripción original y en citas de trabajos referidas al cono sur y al continente europeo, con al menos una ilustración de las especies.

Los ejemplares fueron montados en portaforaminíferos y fotografiados mediante el Microscopio Electrónico de Barrido (SEM) de la Universidad de Concepción.

Los aspectos paleoecológicos que aquí se consideran, se basan en la escasa bibliografía existente sobre foraminíferos terciarios y sobre paleoecología chilena. Las consideraciones personales surgen de criterios ecológicos amplios, y en mayor grado de apreciaciones cualitativas y cuantitativas en el DS1. Estas últimas resultado de la diversidad, expresada como equitatividad (E), dominancia de asociaciones foraminerológicas, relaciones entre grandes grupos de foraminíferos (miliólidos, aglutinados) y entre foraminíferos planctónicos y bentónicos (Tabla 2).

\section{RESULTADOS}

Como resultado de este estudio se presentan las siguientes reconstrucciones paleoecológicas para cada uno de los pisos analizados del pozo DS 1 (Fig. 2 , Tablas 2 y 3), utilizando las especies indicadoras.

GERMANIANO: este piso aparece caracterizado por los siguientes foraminíferos: Praeglobobulimina kickapooensis, Spiroplectammina grzybowski, Anomalinoides cf. nobilis, Glomospira gordialis, Pullenia alazanensis, P. bulloides, Gyroidina soldanii y Cyclammina cancellata. Específicamente en el DS1 el análisis cuantitativo, de la fauna entrega los siguientes datos: 2.250-2.170 m de profundidad de sondaje: 11 especies y 56 ejemplares (4\% planctónicos y $96 \%$ bentónicos), de éstos las formas aglutinadas y calcáreas se encuentran en partes iguales. La diversidad expresada por la equitatividad (E) es igual a 77\% y analizada muestra por muestra varía entre $62 \%$ y $96 \%$.

Los foraminíferos bentónicos Pullenia y Gyroidina son propios de la zona batial, en el talud superior (Boltovskoy 1965) y la frecuencia de bulimínidos indican facies regresivas del mar daniano (Malumián 1978). Sin embargo, la zona del talud superior se caracteriza por tener un bajo 
Tabla 2. Profundidad (Prof.), número y porcentajes de ejemplares de foraminíferos planctónicos (PLANCT) y bentónicos (BENT) e índice de Equitatividad (E), correspondiente Pozo Dorado Sur № 1.

\begin{tabular}{|c|c|c|c|c|c|c|}
\hline № MUESTRA & $\begin{array}{c}\text { PROF. } \\
(\mathrm{m})\end{array}$ & № SPP. & $\begin{array}{c}\text { № } \\
\text { EJEM. }\end{array}$ & $\%$ PLANCT & \% BENT. & $\begin{array}{c}E \\
(\%)\end{array}$ \\
\hline 40 & $750-759$ & 1 & 1 & 0 & 100 & - \\
\hline 42 & $786-795$ & 8 & 108 & 93 & 99 & 66 \\
\hline 43 & $804-813$ & 10 & 96 & 16 & 84 & 65 \\
\hline 44 & $822-831$ & 6 & 15 & 0 & 100 & 90 \\
\hline 45 & $840-849$ & 7 & 15 & 0 & 100 & 93 \\
\hline 46 & $858-867$ & 5 & 9 & 0 & 100 & 95 \\
\hline 47 & $876-885$ & 3 & 4 & 0 & 100 & 95 \\
\hline 48 & $894-903$ & 3 & 3 & 0 & 100 & 100 \\
\hline 49 & $912-921$ & 1 & 2 & 0 & 100 & - \\
\hline 50 & $930-939$ & 3 & 6 & 0 & 100 & 79 \\
\hline 51 & $948-957$ & 6 & 28 & 0 & 100 & 74 \\
\hline 53 & $984-993$ & 2 & 2 & 0 & 100 & 100 \\
\hline 55 & $1018-27$ & 2 & 4 & 0 & 100 & 41 \\
\hline 56 & $1036-45$ & 3 & 7 & 0 & 100 & 87 \\
\hline 57 & $1054-63$ & 4 & 6 & 0 & 100 & 74 \\
\hline 58 & $1072-81$ & 1 & 2 & 0 & 100 & - \\
\hline 60 & $1108-17$ & 10 & 21 & 0 & 100 & 83 \\
\hline 61 & $1120-35$ & 13 & 42 & 0 & 100 & 82 \\
\hline 62 & $1144-53$ & 13 & 33 & 0 & 100 & 83 \\
\hline 63 & $1162-71$ & 11 & 25 & 0 & 100 & 88 \\
\hline 64 & $1180-89$ & 5 & 10 & 0 & 100 & 96 \\
\hline 65 & 1198-07 & 11 & 22 & 0 & 100 & 95 \\
\hline 66 & $1216-25$ & 11 & 17 & 0 & 100 & 96 \\
\hline 67 & $1234-43$ & 15 & 72 & 0 & 100 & 76 \\
\hline 68 & $1252-61$ & 11 & 52 & 0 & 100 & 75 \\
\hline 69 & $1270-79$ & 19 & 87 & 0 & 100 & 87 \\
\hline 70 & $1288-99$ & 13 & 35 & 3 & 97 & 83 \\
\hline 71 & $1306-15$ & 22 & 66 & 3 & 97 & 83 \\
\hline 72 & 1324-33 & 14 & 93 & 0 & 100 & 65 \\
\hline 73 & $1342-51$ & 17 & 58 & 2 & 98 & 75 \\
\hline 74 & $1360-69$ & 12 & 48 & 0 & 100 & 83 \\
\hline 75 & $1378-87$ & 13 & 64 & 0 & 100 & 86 \\
\hline 76 & $1396-05$ & 15 & 75 & 0 & 100 & 80 \\
\hline 77 & $1414-23$ & 17 & 91 & 0 & 100 & 82 \\
\hline 78 & $1432-41$ & 16 & 32 & 0 & 100 & 94 \\
\hline 79 & $1450-59$ & 18 & 42 & 0 & 100 & 96 \\
\hline 80 & $1468-77$ & 19 & 55 & 0 & 100 & 87 \\
\hline 81 & $1486-95$ & 17 & 45 & 2 & 98 & 82 \\
\hline 82 & $1504-13$ & 15 & 27 & 15 & 85 & 92 \\
\hline 83 & $1522-31$ & 23 & 106 & 3 & 97 & 80 \\
\hline 84 & $1540-49$ & 31 & 191 & 9 & 91 & 75 \\
\hline 85 & $1558-67$ & 31 & 253 & 5 & 85 & 74 \\
\hline 86 & $1576-85$ & 24 & 109 & 14 & 86 & 85 \\
\hline 87 & 1594-03 & 20 & 116 & 10 & 90 & 88 \\
\hline 88 & $1612-21$ & 24 & 155 & 12 & 88 & 85 \\
\hline 89 & $1630-39$ & 32 & 187 & 5 & 95 & 81 \\
\hline 90 & $1648-57$ & 27 & 345 & 4 & 96 & 78 \\
\hline 91 & $1666-75$ & 25 & 112 & 5 & 95 & 84 \\
\hline
\end{tabular}


Continuación Tabla 2.

\begin{tabular}{|c|c|c|c|c|c|c|}
\hline № MUESTRA & $\begin{array}{c}\text { PROF. } \\
(\mathrm{m})\end{array}$ & № SPP. & $\begin{array}{c}\text { № } \\
\text { EJEM. }\end{array}$ & $\%$ PLANCT & $\%$ BENT. & $\begin{array}{c}E \\
(\%)\end{array}$ \\
\hline 92 & $1684-93$ & 25 & 286 & 2 & 98 & 80 \\
\hline 93 & $1702-11$ & 23 & 136 & 2 & 98 & 84 \\
\hline 94 & $1720-29$ & 17 & 80 & 1 & 99 & 83 \\
\hline 95 & $1738-49$ & 19 & 47 & 4 & 96 & 94 \\
\hline 96 & $1756-65$ & 10 & 19 & 11 & 89 & 92 \\
\hline 97 & $1774-83$ & 17 & 58 & 7 & 93 & 89 \\
\hline 98 & 1792-01 & 22 & 152 & 4 & 96 & 82 \\
\hline 99 & $1810-19$ & 18 & 85 & 11 & 89 & 76 \\
\hline 100 & $1828-37$ & 22 & 228 & 25 & 75 & 75 \\
\hline 101 & $1846-55$ & 15 & 132 & 19 & 81 & 70 \\
\hline 102 & $1864-73$ & 9 & 37 & 11 & 89 & 86 \\
\hline 103 & $1882-91$ & 8 & 52 & 10 & 90 & 78 \\
\hline 104 & 1900-09 & 17 & 144 & 9 & 91 & 73 \\
\hline 105 & $1918-27$ & 18 & 107 & 8 & 92 & 81 \\
\hline 106 & $1936-45$ & 21 & 249 & 14 & 86 & 85 \\
\hline 107 & $1954-63$ & 18 & 102 & 0 & 100 & 83 \\
\hline 108 & $1972-81$ & 23 & 192 & 12 & 88 & 85 \\
\hline 109 & 1990-99 & 20 & 156 & 26 & 74 & 77 \\
\hline 110 & $2008-17$ & 13 & 45 & 16 & 84 & 86 \\
\hline 111 & $2026-35$ & 5 & 11 & 18 & 82 & 91 \\
\hline 112 & $2044-53$ & 20 & 87 & 46 & 54 & 93 \\
\hline 113 & $2062-74$ & 16 & 84 & 50 & 50 & 83 \\
\hline 114 & $2080-89$ & 13 & 120 & 58 & 42 & 80 \\
\hline 115 & 2089-07 & 15 & 281 & 86 & 14 & 51 \\
\hline 116 & $2116-25$ & 9 & 43 & 40 & 60 & 88 \\
\hline 117 & $2134-43$ & 7 & 19 & 5 & 95 & 90 \\
\hline 118 & 2151-61 & 10 & 22 & 0 & 100 & 81 \\
\hline 119 & $2170-79$ & 4 & 5 & 20 & 80 & 96 \\
\hline 120 & 2188-97 & 2 & 4 & 0 & 100 & 81 \\
\hline 121 & 2197-06 & 3 & 5 & 0 & 100 & 87 \\
\hline 122 & 2210-19 & 5 & 17 & 0 & 100 & 62 \\
\hline 123 & $2224-33$ & 5 & 20 & 5 & 95 & 72 \\
\hline 124 & $2242-50$ & 3 & 5 & 0 & 100 & 96 \\
\hline 125 & $2251-60$ & 7 & 24 & 8 & 92 & 91 \\
\hline
\end{tabular}

porcentaje de ejemplares aglutinados. Esta situación que es contraria a la que se tiene en este piso, en donde la fauna está dominada principalmente por Spiroplectammina (37,5\%), lo cual estaría determinando aguas someras e hiposalinas. Por lo tanto, es posible que la profundidad de las aguas no haya sobrepasado en demasía la zona fótica.

BRUNSWICKIANO: son características de este piso las siguientes especies: Allomorphina aguafrescaensis, A. conica, Elphidium skyringense, E. patagonicum, Spiroplectammina adamsi, Dorothia principensis y Chilostomella cylindroides.
En este piso, el pozo DS1 que va desde los 2.143 a $2.062 \mathrm{~m}$ de profundidad de sondaje se encontraron 29 especies, 570 ejemplares (63\% planctónicos: y 37\% bentónicos:). La diversidad (E) es igual a 78. Las formas aglutinadas disminuyen su porcentaje en comparación con el piso anterior de 50 a 30\% aproximadamente. La presencia del género Elphidium define la existencia de un fondo relativamente bajo, es decir, tipo sublitora1 interno (10-50 m) (Martínez 1954, 1957). La razón planctónico-bentónica es relativamente más alta en este piso. De modo que, es posible que existiera un ambiente de plataforma externa en donde la fauna de 

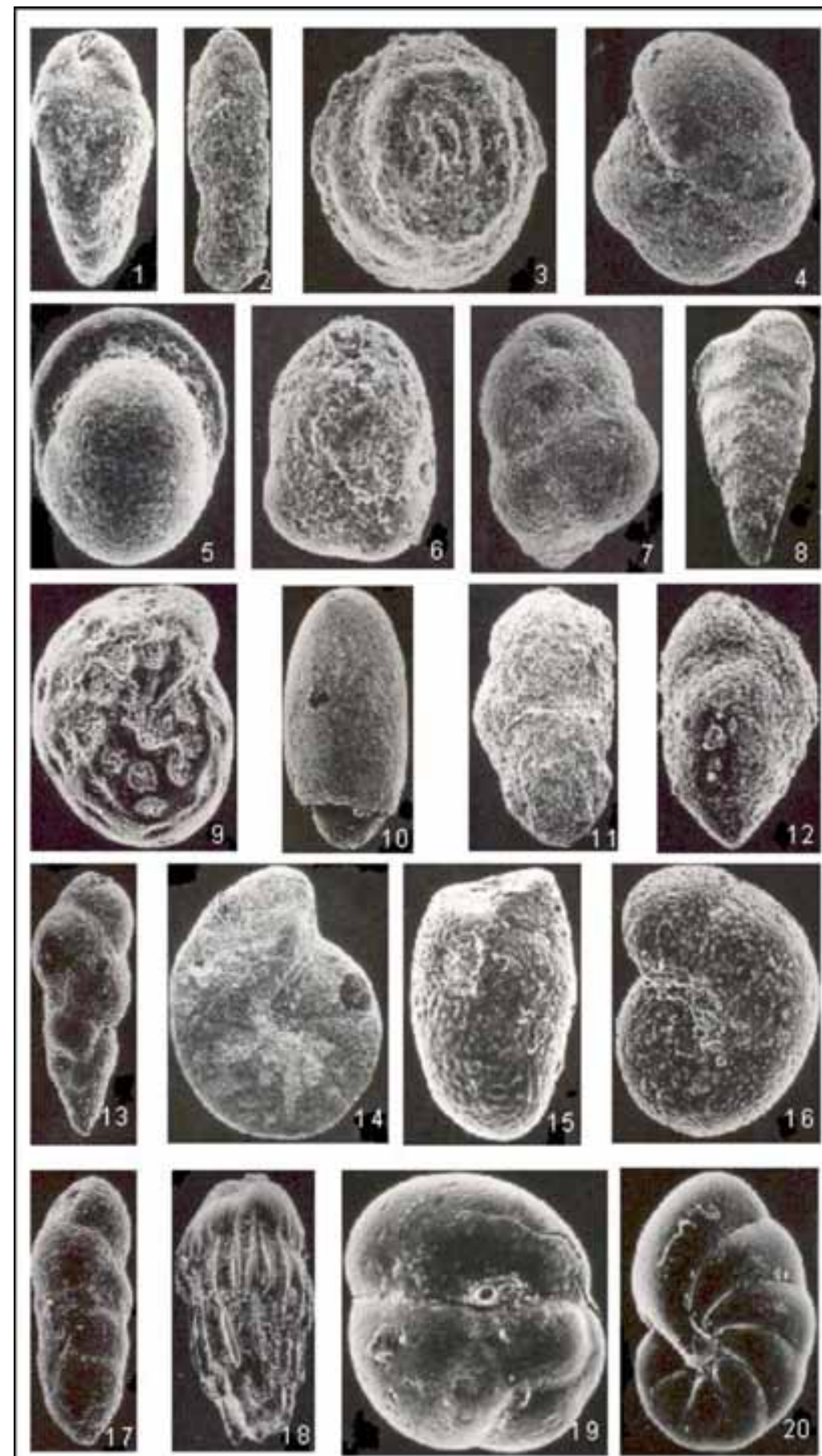

Fig. 2. Especies de foraminíferos bentónicos indicadores más representativos del Pozo Dorado Sur №1 1. Praeglobobulimina kickapooensis, 2. Spiroplectammina grzybowski, 3. Glomospira gordialis, 4. Pullenia alazanensis, 5. P. bulloides, 6. Allomorphina aguafrescaensis, 7. A. conica, 8. Spiroplectammina adamsi, 9. Elphidium saginatum, 10. Chillostomella cylindroides, 11. Plectina elongata, 12. Dorothia principensis, 13. Kolesnikovella severini, 14. Cyclammina cancellata, 15. Boltouskoyella argentinensis, 16. Epistomaroides pinguilabra, 17. Karreriella cushmani, 18. Rectuvigerina ongleyi, 19. Sphaeroidina bulloides, 20. Nonionella auris. 
Tabla 3. Evolución paleobatimétrica documentada mediante foraminíferos bentónicos en cada uno de los Pisos.

\begin{tabular}{|c|c|c|c|}
\hline PISOS & FACIES & & ESPECIES INDICADORAS \\
\hline GAVIOTANO & & & Astrononion sp., N. auris \\
\hline MIRA-ROSARIANO & $\leftarrow$ & & $\begin{array}{l}\text { E. pinguilabra, C. elmaensis, } K . \text { cushmani, Pç bulloides, S. bulloides, } \\
\text { R. ongleyi, B. argentinensis, S. gracilis, M. communis }\end{array}$ \\
\hline CAMERONIANO & & $\rightarrow$ & $\begin{array}{l}\text { E. saginatum, C. cf. parki, } P \text {. wuellerstorfi, Marginulina spp., } N \text {. } \\
\text { cf. commune, K. severini, Cassidulina sp., Pyrgo sp., Cibicides sp., } \\
\text { las familias Buliminidae, Lagenidae y de las formas aglutinadas: } C \text {. } \\
\text { cancellata, M. communis, Haplophragmoides sp. }\end{array}$ \\
\hline MORITZIANO & & & $\begin{array}{l}\text { P. elongata, S. cf. brunswickensis, E. hampdenense y de las familias } \\
\text { Buliminidae y Lagenidae. Acompañados de radiolarios. }\end{array}$ \\
\hline CLARENCIANO & $\leftarrow$ & $\overline{-\cdots}$ & C. cylindroides, D. principensis, E. hampdenense. \\
\hline BRUNSWICKIANO & & - & $\begin{array}{l}\text { A. aguafrescaensis, A. conica, E. skyringense, E. patagonicum, S. } \\
\text { adamsi, D. principensis, C. cylindroides. }\end{array}$ \\
\hline GERMANIANO & & & $\begin{array}{l}\text { P. kickapooensis, S. grzybowski, A. cf. nobilis, G. gordialis, P. ala- } \\
\text { zanensis, P. bulloides, G. soldanii, C. cancellata. }\end{array}$ \\
\hline $\begin{array}{ll}\longleftrightarrow & \text { Transgresión } \\
& \text { Regresión }\end{array}$ & $\begin{array}{l}\text { Plataforma } \\
\text { Interna }\end{array}$ & $\begin{array}{l}\text { Plataforma } \\
\text { Externa }\end{array}$ & \\
\hline
\end{tabular}

foraminíferos es variada. Las especies planctónicas sugieren una plataforma continental estrecha y un aumento brusco de la profundidad.

CLARENCIANO: el piso se caracteriza por la presencia de Chillostomella cylindroides, Dorothia principensis y Elphidium hampdenense.

Los sedimentos de este piso se encuentran muy poco desarrollados debido a que no cubren más allá de $10 \mathrm{~m}$ (2.053-2.044 $\mathrm{m}$ de profundidad de sondaje, 1 muestra $\mathrm{N}^{\circ} 112$ ).

En el Pozo DS1 existen 20 especies y 87 ejemplares (47\% bentónicos y 53\% planctónicos). Se mantiene la razón planctónico-bentónica aproximadamente cercana a la unidad, lo que permite determinar condiciones paleoecológicas similares al piso anterior. La diversidad (E) es igual a 93.

MORITZIANO: los microfósiles diagnósticos son Plectina elongata, Spiroplectammina cf. brunswickensis. Acompañados de gran cantidad de radiolarios que aparecen en este piso.

$\mathrm{Al}$ grupo de las especies predominantes se les suma Elphidium hampdenense y las familias Buliminidae y Lagenidae demostrándose que la fauna de foraminíferos se hace visiblemente más variada y rica cuantitativamente, característica de la zona de aguas de la plataforma interna.

En el Pozo DS1: los sedimentos se encuentran entre los 2.035 a los $1.864 \mathrm{~m}$ de profundidad de sondaje. Se obtuvieron 44 especies, con 1.097 ejemplares (13\% planctónicos y $87 \%$ bentónicos). La diversidad (E) es igual a 72 y muestra por muestra varía entre 73 y $91 \%$. El porcentaje de formas aglutinadas no alcanza al $5 \%$ y las especies planctónicas se encuentran como excepción.

Los radiolarios spumeláridos presentes en la columna predominan entre las 25 y 150 brazas (45-270 m de profundidad) lo que correspondería a un ambiente netamente oceánico muy cercano al límite epi-mesopelágico (Cañón 1968a).

CAMERONIANO: los sedimentos atribuidos a este piso contienen las especies Elphidium saginatum, Cibicides cf. parki, Planulina wuellerstorfi, Marginulina sp. A, Marginulina sp. B, Nonion cf. commune y Kolesnikovella severini, Cassidulina sp., Pyrgo sp., Cibicides sp. Además, se encuentran representantes de las familias Buliminidae, Lagenidae y de las formas aglutinadas Cyclammina cancellata, Martinotiella communis y Haplophragmoides sp.

Específicamente en el Pozo DS1: los sedimentos cubren desde los 1.855 a los $1.594 \mathrm{~m}$ de profundidad de sondaje, se obtuvo 60 especies con 2.130 ejemplares (8\% planctónicos y 92\% bentónicos). Las formas aglutinadas llegan al 7,5\% del $92 \%$ y la equitatividad es igual al $75 \%$, variando entre 70 y $94 \%$.

La fauna determinada es propia de una plataforma externa principalmente por las especies del 
género Cibicides, además de Nonion, Cassidulina, Pyrgo y los aglutinados Bigenerina y Martinotiella.

ROSARIANO-MIRADORIANO: las especies típicas bentónicas de estos pisos son: Epistomaroides pinguilabra, Cibicides elmaensis, Karreriella cushmani, Pullenia bulloides, Sphaeroidina bulloides, Rectuvigerina ongleyi, Boltovskoyella argentinensis y las formas aglutinadas Spiroplectammina gracilis y Martinotiella communis.

En el Pozo DS1 el piso que abarca desde los 1.585 a los $822 \mathrm{~m}$ de profundidad de sondaje, con un total de 1.884 ejemplares distribuidos en 68 especies, principalmente bentónicas. Las especies planctónicas se encuentran en muy pequeña cantidad (3\%). Posee una equitatividad (E) igual a $76,3 \%$, variando entre 100 y $74 \%$. A esta fauna se agregan algunos representantes de las familias Miliolidae, Buliminidae y del género Nonionella típico de bahías internas.

Esta asociación determina aguas de plataforma interna, debido a la presencia de Pullenia, Sphaeroidina, Lenticulina y las formas aglutinadas Martinotiella y Haplophragmoides.

GAVIOTIANO: son características de este piso las especies Astrononion sp. y Nonionella auris. En el Pozo DS1 los sedimentos de este piso abarcan desde los 822 hasta los $678 \mathrm{~m}$ de profundidad de sondaje. Se encuentra un $70 \%$ de equitatividad, variando entre 66 y 65\%, con 205 ejemplares y 12 especies, en donde los foraminíferos planctónicos constituyen aproximadamente un $1 \%$, los bentónicos el 99\%. Las formas aglutinadas representan un 9\% del total.

El género Nonionella posee un $36 \%$ de representación en este piso y es el taxón que estaría determinando un ambiente de plataforma interna.

\section{DISCUSIÓN Y CONCLUSIONES}

Las especies bentónicas constituyen un buen medio para descifrar y reconstruir detalles del paleoambiente (Cañon 1968a; Boltovskoy 1980a). El uso de la relación planctónica-bentónica como indicador batimétrico fue introducido por Grimsdale \& Van Morkhoven (1955) y Smith (1955) (fide Malumián 1978). Sin embargo, trabajos posteriores han puntualizado que estas estimaciones pueden llevar a conclusiones erróneas, debido al hecho de que la abundancia del plancton depende no sólo de la profundidad sino también de la salinidad, luz, nutrientes y otros parámetros abióticos del ambiente marino (Malumián 1978).

Se ha observado que no es discernible la variable "distancia a la costa"; al respecto Murray \& Hawkins (1976) han propuesto un método basado en el tamaño de los foraminíferos planctónicos, pero hasta el momento no hay datos concretos de su factibilidad.

La investigación de la posible influencia de las variables químicas del sedimento de fondo sobre los microorganismos bentónicos es un aspecto importante de la ecología (Boltovskoy 1965). Sin embargo, existe en la actualidad una carencia casi total de estudios sobre el tema.

Por lo tanto, las ideas ecológicas determinadas deben considerarse con la debida reserva, ya que en su mayoría son obtenidas de otras cuencas muy distintas. Se ha propuesto que la fauna que constituye las diferentes etapas o pisos tiene sus homeomorfos que viven en los océanos actuales.

De esta manera, se puede discutir las dos ingresiones (Tabla 3) definidas por las interpretaciones obtenidas; así durante el Germaniano (edad eocena temprana-paleocena temprana) se habría estado produciendo un ascenso y somerización de la cuenca, explicitado por la presencia del género Spiroplectammina que indicaría aguas someras e hiposalinas, concordando así con Malumián (1978) y Cañón (1968a).

En el Bruswickiano (edad eoceno temprana-paleocena tardía) se habrían restablecido las condiciones normales de salinidad, debido a la ingresión de las aguas con profundidades de $200 \mathrm{~m}$ aproximadamente (plataforma externa); las formas planctónicas alcanzan aquí su mejor representación. Estas condiciones paleoecológicas se habrían mantenido durante el Clarenciano (edad eocena media temprana), lo que vendría a corresponder a la parte inferior del Parafiniano de Cañón (1968a).

La disminución de las formas planctónicas, la presencia de Elphidium y la Familia Buliminidae indican aguas de plataforma interna durante el Moritziano (edad eocena media superior).

Una segunda ingresión durante el Cameroniano (edad eocena tardía) (parte inferior del Concordiano de Cañón, 1968) sería la causa de la presencia de los géneros Cibicides, Cassidulina, Pyrgo, Bigenerina y Martinotiella. 
Durante el Rosariano-Miradoriano (edad eocena tardía-oligocena temprana) la especie Boltovskoyella argentinensis, las formas aglutinadas Spiroplectammina gracilis, Karreriella cushmani y las familias Miliolidae y Buliminidae conformarían una asociación típica de plataforma interna en el área de estudio (Cuenca de Magallanes) y estas mismas características se habrían registrado en la parte argentina (Malumián 1978). Finalmente en el Gaviotiano (edad oligocena temprana) las condiciones serían las mismas del piso anterior, principalmente por la dominancia del género $\mathrm{No}^{-}$ nionella y los escasos foraminíferos planctónicos. Lo que se enmarca dentro de lo encontrado por Cañón (1968a, p. 39).

\section{AGRADECIMIENTOS}

A la Empresa Nacional del Petróleo (ENAPMagallanes), que permitió el viaje y estadía en Punta Arenas, durante el período de análisis de las muestras (1991). Al Sr. Vicente Pérez-D’Angello, Jefe del Departamento de Protección Ambiental, por las facilidades y bibliografía otorgadas en ese entonces.

Al Laboratorio de Micropaleontología del Instituto de la Patagonia de la Universidad de Magallanes, donde me facilitaron las colecciones micropaleontológicas de referencia y muestras, espacio físico, material óptico y bibliográfico. A la Prof. Tatiana Hromic M. por su constante preocupación y oportunos aportes al buen desarrollo de la investigación.

A los profesores Hugo Moyano G. y Víctor H. Ruiz R., del Departamento de Zoología, Universidad de Concepción, por su disposición, así como sus revisiones y sugerencias críticas del manuscrito. $\mathrm{Al}$ personal técnico del Laboratorio de Microscopia Electrónica de la Universidad de Concepción por las fotografías.

Este trabajo es una contribución al proyecto SCOR (Scientific Committee on Oceanic Research) Working Group 138 membership.

\section{LITERATURA CITADA}

Bandy, O. \& R. Arnal 1960. Concepts of Foraminiferal Paleoecology. Bulletin of the American Association of Petroleum Geologist, 44(12): 1921-1932, 14 figs.
Bermúdez, J. \& H. Gómez 1966. Estudio Paleontológico de una Sección del Eoceno. Grupo Punta Carnero de la Isla Margarita, Venezuela. Memorias de la Sociedad de Ciencias Naturales La Salle, XXVI (75): 205-259

Boltovskoy, E. 1965. Los Foraminíferos Recientes (Biología, métodos de estudio, aplicación oceanográfica). EUDEBA, Buenos Aires: $510 \mathrm{pp}$.

Boltovskoy, E. 1980a. Perforación Gil 1, Provincia de Buenos Aires (Foraminíferos, Edad, Paleoambiente). Ameghiniana, 17(4): 339-362

Boltovskoy, E. 1980b. Foraminíferos Bentónicos de la Zona Batial Media como Fósiles-guías en Depósitos Oligogénicos-Cuartarios de los Océanos Pacífico, Atlántico e Indico. In: Actas 2 o Congreso Argentino de Paleontología y Bioestratigrafía y $1^{\circ}$ Congreso Latinoamericano de Paleontología, 2: 341-362

Brady, H. 1879. Notes on some of the Reticularian Rhizopoda of the "Challenger Expeditions". Quarternary Journal Micropaleontology Science Serie, 19: 261-299, 8 pls.

Brotzen, F. 1948. The Swedish Paleocene and its Foraminiferal Fauna. Sveriges Geologiska Undersokning. Serie C. № 493: 1-140, pls. 1-19, text. y figs. 1-41, table. 1.

Cañón, A. 1968a. Cronoestratigrafía de los Sedimentos Terciarios de Tierra del Fuego, Provincia de Magallanes. Memoria para optar al Título de Geólogo. U. de Chile. Facultad Ciencias Facultad y Matemáticas Departamento Geología, 126 pp, 7 láms.

Cañón, A. 1968b. Cronoestratigrafía de los Sedimentos Terciarios de Tierra del Fuego, Provincia de Magallanes. Tercera Jornadas Geológicas Argentinas, Comodoro Rivadavia, 1: 91-110.

Cushman, J. 1927. Some characteristic Mexican Fossil Foraminifera. Journal Paleontology, I: $147-172$, pls. 23-28.

Cushman, J. 1929. The Foraminifera of the Atlantic Ocean. Part. 6 Miliolidae, Ophthalmidiidae and Fischerinidae. Smithsonian Institution United States National Museum Bulletin, 104: 129, 22 pls.

Cushman, J. \& P. Bermúdez 1936. Additional new species of foraminifera and a new genus from the Eocene of Cuba. Cushman Laboratory Research Contribution, 12: 55-63, pls. 10-11. 
Cushman, J \& R. Todd 1949. Species of the genera Allomorphina and Quadrimorphina. Cushman Foundation Foramimiferal Research Contribution, 25(3): 59-72, pls. 11 y 12.

Finlay, H. 1939. New Zealand Foraminifera: key species in stratigraphy № 2. Royal Society New Zealand Transactions Proceedings, 69(1): 89-128, pls. 11-14.

Finlay, H. 1940. New Zealand Foraminifera: key species in stratigraphy №4 69(4). Royal Society New Zealand Transactions Proceedings, 68(1): 504-533, pls. 68-69, 69: 448-473, pls. 11-14.

Hromic, T. 1990. Lista Preliminar de foraminíferos planctónicos terciarios de la porción chilena de la cuenca Austral. Actas $2^{\circ}$ Simposio sobre el Terciario de Chile. Departamento de Geociencias, Facultad de Ciencias, Universidad de Concepción, Concepción, 1 pp.

Loeblich, A. \& H. Tappan 1964. Part C. Protista 2 In: Treatise of Invertebrate Paleontolog. University of Kansas Press, Lawrence Kansas, Ed. Moore. XXXI + 900 s., 653 ABB.

Loeblich, A. \& H. Tappan 1988. Foraminiferal Genera and Their Classification. Van Nostrand Reinhold Co. New York, text-vol. 970 pp., pl-vol: 212 pp. +847.

Malumián, N. 1978. Esbozo Paleoecológico de las Asociaciones Foraminiferológicas Terciarias de la Argentina. Ameghiniana, 15(1-2): 161-171.

Malumian, N. \& V. Masiuk 1972. Boltouskoyella: A new Paleogene Foraminiferal Genus from Argentina. Journal of Foraminiferal Research, 2(1): $1-5,1 \mathrm{pl}$.

Malumián, N. \& C. Náñez 1989. Asociaciones de foraminíferos del Terciario Medio de la Cuenca Austral: sus relaciones con eventos eustáticos globales. Revista de la Asociación Geológica, 42(2): 257-264.

Marchant, M. 1998. Zonación mediante foraminíferos planctónicos del Paleógeno del área Dorado-Kimiri Aike Sur, de la Cuenca de Magallanes, Chile. Revista Española de Micropaleontología, 30(3): 49-57.

Martínez, R. 1954. Revisión de las Especies de Elphidium de la Cuenca de Magallanes (Informe ENAP, inédito, sin № de pp.).

Martínez, R. 1957. El Terciario Superior en Algunos Pozos del Continente de Tierra del Fuego (Informe ENAP, inédito, sin № de pp.).
Martínez, R. 1968. Zonación Preliminar del Terciario de Chile Central, mediante foraminíferos Planctónicos y su Correlación Regional y Transcontinental. In: Ceccioni G. (Ed.) El Terciario de Chile Zona Central. Sociedad Geológica de Chile. Ed. Andrés Bello, 95-102.

Murray, J. \& A. Hawkins 1976. Sediments Transport in the Severn Estuary during the Past 8000-9000 Years. Journal Geology Society London, 132(4): 385-398, 5 figs. 1 pl.

Natland, M. \& E. González. 1965. A System of Stages for Correlation of Magallanes Basin Sediments (Informe ENAP, inédito): 1-18.

Natland, M., E. González, A. Cañón \& M. Ernst 1974. A System of Stages for Correlations of Magallanes Basin Sediment. Geological Society America Memoir, 139: 1-117, 6 pls.

Orbigny, A, d'. 1826. Tableau méthodique de la Clase des Cephalopodes. Annals Science Natural, 7: 245-314, pls. 10-17.

Rau, W. 1948. Foraminifera from the Porter Shale (Lincoln Formation), Grays Harbor County. Washington Journal Paleontology, 22: 1-173.

Reuss, A. 1851. Uber die fossilen Foraminiferen und Entomostracen der Septarienthone der Umgegend von Berlin. Zeitschr. Deutsch. Geol. Ges., 3: 49-92, pls. 3-7.

Todd, R. \& H. Kniker 1952. An Eocene Foraminiferal Fauna from the Agua Fresca Shale of Magallanes Province, Southernmost, Chile. Cushman Foundation Foraminiferal Research Special Publication, 1: 28, 4 pls., 4 figs.

\section{LISTA TAXONÓMICA DE REFERENCIA}

Allomorphina aguafrescaensis Todd \& Kniker, 1952: 25, pl. 4. fig. 31.

Allomorphina conica Cushman \& Todd, 1949: 62, pl. 11 , fig. 8 .

Anomalinoides cf. nobilis. Anomalinoides nobilis. Brotzen, 1948: 89, lám. 14, fig. 5.

Boltouskoyella argentinensis Malumian \& Masiuk, 1972: 2-3, pl.1, figs. 3-6.

Chilostomella cylindroides Reuss, 1851: 80, pl. 6, fig. 43.

Cibicides elmaensis Rau, 1948: 173.

Cibicides cf. parki. Cibicides parki Finlay, 1939, 68: 528, lám. 69, fig. 1.

Cyclammina cancellata Brady, 1879: 62, s. 19. 
Dorothia principensis Cushman \& Bermudez, 1936: 57, pl. 10, figs. 3 y 4 .

Elphidium hampdenense Finlay, 1940: 457. 16 M. Marchant

Elphidium patagonicum Todd \& Kniker, 1952: 18 pl. 3, fig. 35 a, b.

Elphidium saginatum Finlay, 1940: 457.

Elphidium skyringense Todd \& Kniker, 1952: 18, pl. 3, fig. 39 a y b.

Epistomaroides pinguilabra (= Anomalina pinguilabra Finlay, 1940): 460.

Glomospira gordialis (Jones \& Parker, 1860): 30 (fide Cañón, 1968a: 73).

Gyroidina soldani d'Orbigny, 1826: 276, № 5, mod. 36.

Karreriella cushmani Finlay, 1940: 452, pl. 63, figs. 38-42.

Kolesnikovella severini (Cañón \& Ernst). Malumián \& Nañez, 1989: 257.

Martinotiella communis (d'Orbigny). Loeblich \& Tappan. 1964: C282, fig. 188-10, 188-11.

Nonion cf. commune (d'Orbigny). Cañón, 1968a: 91, lám. 3, figs. $30 \mathrm{a}, \mathrm{b}$.
Planulina wuellerstorfi (Schwager). Cushman, 1929: 104, pl. 15, figs. 1 y 2.

Plectina elongata Cushman \& Bermudez, 1936: 58 , pl. 10, figs. 22-24.

Praeglobobulimina kickapooensis (Cole). Natland et al., 1974: 77, pl. 2, figs. 13 a-c.

Pullenia alazanensis Cushman, 1927: 168, pl. 26, fig. 14 y 15.

Pullenia bulloides (d’Orbigny). Bermudez \& Gómez, 1966: 240, lám 6, figs. 9 y 10.

Rectuvigerina ongleyi (Finlay). Cañón 1968a: 104, lám. 4, fig. 52 a, b.

Sphaeroidina bulloides d'Orbigny, 1826: 267, № 65.

Spiroplectammina adamsi Lalicker, 1935: 39, pl. 6, fig. 1,2. (fide Todd \& Kniker, 1952).

Spiroplectammina gracilis (Von Muenster). Cañón, 1968a: 75, lám. 1, figs. 7 a, b y c.

Spiroplectammina grzybowski Frizzell, 1928: 101, lám. 14, fig. 13 y 14 (fide Cañón, 1968a).

Spiroplectammina cf. brunswickensis. Spiroplectammina brunswickensis Todd \& Kniker, 1952: 6, pl. 1, fig. 16 